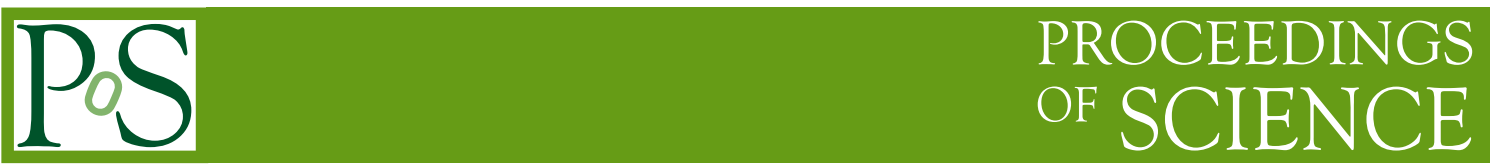

\title{
Parsec-scale Nuclear Radio Structures in Seyfert Galaxies
}

\section{Preeti Kharb*}

National Centre for Radio Astrophysics - Tata Institute of Fundamental Research (NCRA-TIFR), S. P. Pune University Campus, Post Bag 3, Ganeshkhind, Pune 411007, India

E-mail: kharbancra.tifr.res.in

\begin{abstract}
Radio outflows of extents ranging from a few parsecs to a few kiloparsecs are present in Seyfert and LINER galaxies that make up the "radio-quiet" AGN class. AGN jets and / or starburst superwinds have been suggested to produce these outflows. We present a brief review of radio outflows in Seyfert and LINER galaxies on different spatial scales. Very long baseline interferometry (VLBI) observations of several individual Seyferts and LINERs suggest a link between AGN jets on parsec-scales and their kiloparsec-scale radio structures (KSRs). The whole range of misalignment angles present between the parsec-scale and the kpc-scale outflows in Seyfert galaxies and LINERs, supports the prevalence of bent outflows in them. Episodic AGN activity is suggested by the presence of multiple misaligned KSRs in several Seyfert galaxies in total and polarized intensity images; this latter result provides further support for an AGN jet origin of the KSRs present in Seyfert and LINER galaxies.
\end{abstract}

Revisiting narrow-line Seyfert 1 galaxies and their place in the Universe - NLS1 Padova

9-13 April 2018

Padova Botanical Garden, Italy

${ }^{*}$ Speaker. 


\section{Introduction}

Radio outflows of extents ranging from tens to hundreds of parsecs on one end, and ten to twenty kiloparsecs (kpc) on the other, have been detected in Seyfert and LINER galaxies [1-6]. The origin of these radio outflows is not unequivocally clear. [7] and [2] have suggested that these outflows could be powered by starburst superwinds, while [8] have pointed out to the distinct morphological differences between Seyfert outflows and those in starburst galaxies, supporting an AGN origin for the former. [4] have concluded from a VLA study of a complete sample that most kiloparsec-scale radio structures (KSRs) in Seyferts and LINERs are AGN-driven, but the starburst superwind contribution cannot be ruled out. It is worth noting here that while Narrow-line Seyfert 1 galaxies (NLS1s) differ from regular Seyferts in their emission-line and (possibly) black hole properties, they exhibit KSRs similar to the ones observed in Seyferts and LINERs [9, 10].

One of the best ways to probe the AGN role is by studying the radio outflows in Seyferts and LINERs on parsec-scales via the technique of Very Long Baseline Interferometry (VLBI). VLBI arrays have angular resolutions ranging from a few milli-arcseconds (mas, e.g., VLBA ${ }^{1}$ ) to tens and hundreds of mas (e.g., MERLIN ${ }^{2}$ ). For the nearby galaxies, this translates to spatial scales of a few parsecs to a few sub-kiloparsecs (sub-kpc), respectively. Continuous tracing and accounting for the radio contribution going from parsec to sub-kpc to kpc-scales, can go a long way in settling the AGN versus the starburst superwind debate for Seyfert and LINER outflows.

VLBI observations of several Seyfert galaxies have revealed the presence of weak radio cores and one-sided or two-sided radio jets in them $[11,12]$. The brightness temperatures $\left(T_{b}\right)$ of the radio cores are typically of the order of $10^{6}-10^{11} \mathrm{~K}$; they exhibit flat or inverted spectral indices [13]. However, steep spectrum radio "cores" have also been reported in several Seyfert galaxies [14-16]. These "cores" could be contaminated by the presence of steep spectrum jet emission. Alternately, the real radio cores may have failed detection at the observing frequencies and could show up at higher radio frequencies. Overall, the VLBI results are consistent with Seyferts and LINERs harbouring low luminosity AGN [17-19].

\subsection{Probing the Parsec-scale - Kiloparsec-scale Radio Connection}

VLBI jets in several Seyfert galaxies reveal bends and wiggles [14, 20, 21]. Jet precession has been invoked to explain these morphological peculiarties [e.g., 22, 23]. Jet precession in turn could arise due to accretion disk warping, jet instabilities, or the presence of binary black holes. Mrk 6 is one such Seyfert galaxy showing an S-shaped radio jet in MERLIN observations [24]. It is a peculiar galaxy in that it shows two sets of KSRs aligned nearly perpendicular to each other [25]. Our two-frequency $(1.6,5 \mathrm{GHz})$ VLBA observations of Mrk 6 detected an inverted spectrum radio core at the higher frequency for the first time in this source, and resolved the knots in the S-shaped MERLIN jet into elongated jet-like features [26]. The precessing jet model of [27] could fit the parsec-scale emission as well as the bright edges of the north-south oriented KSR. Two episodes of AGN jet activity with precessing jets could explain the entire complex structure observed in Mrk 6 .

A precessing radio jet could also explain the radio emission on parsec-, sub-kpc- and kpcscales in the Seyfert / LINER galaxy with an ongoing nuclear starburst, NGC 6764 [15]. Inter-

\footnotetext{
${ }^{1}$ Very Long Baseline Array

${ }^{2}$ Multi-Element Radio Linked Interfermeter Network
} 
estingly, the precession model best-fit values of jet inclination $\left(\sim 18^{\circ}\right)$ and jet speed $(\sim 0.028 c)$ could explain the observed jet-to-counterjet surface brightness ratio $\left(R_{J} \sim 1.2\right)$ in NGC 6764. We examined the starburst-wind contribution to the radio emission in NGC 6764 and another Seyfert + starburst composite galaxy, NGC 3079 [see 28]. We found that only about 25 - 30\% of the total radio flux density appears to arise in clear equatorial emission that could be attributable to stellar winds, in both these sources. VLBI observations of NGC 3079 have revealed multiple misaligned jet-like features, which are not all consistently along one position angle with respect to the KSR; this lead [29] to invoke a wide-angle parsec-scale outflow in NGC 3079 [see also 30]. In Mrk 6 and NGC 6764 and possibly NGC 3079, precessing jets launched from their black hole - accretion disk systems, could be powering the KSRs and dissipating on sub-kpc or kpc-scales. This could also be the case in the Seyfert galaxies NGC 1320 and NGC 2992: newly acquired eMERLIN data on these sources reveal $\sim 20-30$ parsec-scale core-jet structures in them, that are misaligned to their KSRs (Kharb et al. 2018a, in preparation). Jet precession has also been invoked from direct multi-epoch VLBI observations in the Seyfert / LINER galaxies III Zw 2 and M81 by [31] and [32], respectively.

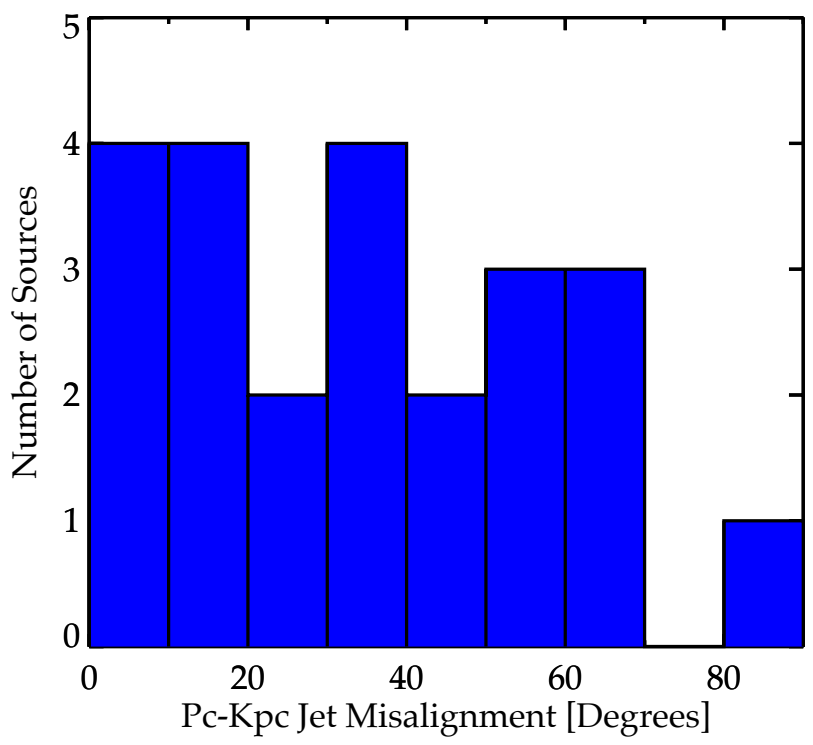

Figure 1: Jet misalignment angles from parsec to kiloparsec scales in Seyfert and LINER galaxies.

In Figure 1 we have plotted the parsec-to-kpc-scale jet misalignment angles in Mrk 6, NGC 6764, NGC 1320, and NGC 2992, along with others from the literature [2, 23]. While there could be large uncertainties in deriving the KSR position angles due to its diffuse structure (of the order of $10^{\circ}-20^{\circ}$ ), we note that the distribution of misalignment angles in Seyferts does not show any clear preference, unlike what is observed in blazars which show a bimodal distribution in misalignment angles $[15,33]$. This latter result has been attributed to the presence of low-pitch helical jets in a sub-population of blazars by [33]. Seyfert outflows on the other hand, show the entire range of misalignment angles. Could continuously bending outflows, that are observationally sampled on different spatial scales in different sources, produce this misalignment angle distribution ? Clearly, many more Seyfert and LINER galaxies need to be examined on several different spatial scales to answer this question. 
Changes in the jet propagation direction from parsec to kpc-scales have often been observed in jetted AGN, as has episodic AGN activity [e.g., 34]. Assuming that jet re-orientation is more likely than re-orientation of large-scale galactic disks producing starburst superwinds, the existence of multiple misaligned KSRs would support an AGN origin for them. Interestingly, secondary misaligned KSRs have been revealed in polarized emission in the Seyfert galaxies NGC 2992 and NGC 3079 in the Continuum Halos in Nearby Galaxies - an EVLA Survey (CHANG-ES) by [35]. These secondary KSRs have not been observed in total intensity images, unlike the case of Mrk 6. Nevertheless, they suggest that episodic AGN activity may be a common phenomena in Seyfert and LINER galaxies.

\subsection{Could Jet Precession Point to the Presence of Binary Black Holes?}

About $1 \%$ of SDSS AGN show double-peaked emission lines in their optical spectra [36]. The presence of double-peaked narrow emission lines in these double-peaked AGN (DPAGN) has been suggested to arise due to (i) binary black holes with associated narrow-line regions (NLRs), (i) jet-NLR interaction or (iii) disky NLRs. In order to search for binary black holes in Seyferts, we have been observing Seyfert DPAGN with phase-referenced VLBI. Dual frequency VLBA observations have detected a single weak $\sim 0.7 \mathrm{mJy}$ radio core of size $(8 \times 6 \mathrm{pc})$ in the Seyfert DPAGN KISSR 1494 [37]. The brightness temperature of this core $\left(\mathrm{T}_{b} \sim 1.4 \times 10^{7} \mathrm{~K}\right)$ and steep radio spectrum $\left(\alpha \leq-1.5 \pm 0.5 ; S_{v} \propto v^{\alpha}\right)$, are consistent with optically thin synchrotron emission. However, the core is not "compact" and its brightness is not centrally concentrated; it gets resolved out in images with different weighting schemes. And yet it does not resemble a typical parsec-scale jet component. We have suggested this component to be the base of a tenuous synchrotron-emitting coronal wind. This could explain the double-peaked narrow lines as also originating in a winddriven NLR.

Similar dual-frequency phase-referenced VLBA observations of the Seyfert DPAGN KISSR 1219, have revealed a one-sided $\sim 70$ parsec radio jet with a steep radio spectrum $[\alpha \leq-1.0 \pm 0.2 ; 38]$. Using the jet-to-counterjet surface brightness ratio in KISSR 1219 and an orientation angle consistent with its Seyfert type 2 classification $\left(\theta \gtrsim 50^{\circ}\right.$ based on the typical dusty torus half opening angle), we concluded that the jet speed had decreased from $\gtrsim 0.55 c$ on parsec-scales to $\gtrsim 0.25 c$ on kpc-scales. The radio jet was likely pushing the NLR clouds in opposite directions, giving rise to the double-peaked emission lines. This may have resulted in jet deceleration and eventual dissipation.

New VLBA observations of the Seyfert DPAGN KISSR 434 have revealed an intriguing 150 parsec C-shaped curved jet, again suggesting jet-NLR interaction as the source of doublepeaked narrow emission lines (Kharb et al. 2018b, in preparation). However, the curved jet itself could arise due to precession in a binary black hole system [e.g., see 39, 40]. Interestingly, we have found suggestions of a binary black hole system through multi-frequency VLBA observations of the Seyfert / luminous infrared galaxy (LIRG) NGC 7674 [41]. This galaxy possesses a Z-shaped $\sim 0.7 \mathrm{kpc}$ radio jet [42]. The dual VLBA cores have $\mathrm{T}_{b} \sim 2-6 \times 10^{7} \mathrm{~K}$ and inverted spectral indices, consistent with being the synchrotron self-absorbed bases of two jets launched from two accreting supermassive black holes. Their projected separation of $\sim 0.65$ mas $(=0.35$ parsec) makes them the closest separation binary black hole pair to be imaged with VLBI. 


\section{Summary}

Radio outflows are frequently observed in Seyfert and LINER galaxies, inspite of their "radioquiet" AGN status. These can span extents ranging from tens of parsecs to $10-20 \mathrm{kpc}$ or more. Nuclear starburst wind contributions to the radio emission cannot be completely ruled out, in at least some Seyferts and LINERs. However, sensitive or phase-referenced VLBI observations that can probe faint parsec-scale radio emission, more often than not reveal weak radio cores and wiggly radio jets in Seyfert and LINER galaxies. In individual sources with multi-scale data on parsec, subkpc and kpc-scales, an AGN jet, which could be curved in many cases, can connect the emission on different spatial scales, making the case for an AGN origin for the radio outflows. Curved jets could suggest jet-ISM interaction or precession, which in turn could suggest the presence of binary black holes or accretion disk instabilities.

\section{Acknowledgements}

We thank the anonymous referee for a positive response to our paper. This conference has been organized with the support of the Department of Physics and Astronomy "Galileo Galilei", the University of Padova, the National Institute of Astrophysics INAF, the Padova Planetarium, and the RadioNet consortium. RadioNet has received funding from the European Union's Horizon 2020 research and innovation programme under grant agreement No 730562.

\section{References}

[1] J. S. Ulvestad, A. S. Wilson and R. A. Sramek, Radio structures of Seyfert galaxies. II, ApJ 247 (1981) 419.

[2] S. A. Baum, C. P. O’Dea, D. Dallacassa, A. G. de Bruyn and A. Pedlar, Kiloparsec-Scale Radio Emission in Seyfert Galaxies: Evidence for Starburst-driven Superwinds?, ApJ 419 (1993) 553.

[3] A. Thean, A. Pedlar, M. J. Kukula, S. A. Baum and C. P. O'Dea, High-resolution radio observations of Seyfert galaxies in the extended 12- $\mu \mathrm{m}$ sample - I. The observations, MNRAS 314 (2000) 573.

[4] J. F. Gallimore, D. J. Axon, C. P. O'Dea, S. A. Baum and A. Pedlar, A Survey of Kiloparsec-Scale Radio Outflows in Radio-Quiet Active Galactic Nuclei, AJ 132 (2006) 546 [astro-ph/0604219].

[5] P. Kharb, S. Srivastava, V. Singh, J. F. Gallimore, C. H. Ishwara-Chandra and H. Ananda, A GMRT study of Seyfert galaxies NGC 4235 and NGC 4594: evidence of episodic activity?, MNRAS 459 (2016) 1310 [1603.08364].

[6] R. D. Baldi, D. R. A. Williams, I. M. McHardy, R. J. Beswick, M. K. Argo, B. T. Dullo et al., LeMMINGs - I. The eMERLIN legacy survey of nearby galaxies. 1.5-GHz parsec-scale radio structures and cores, MNRAS 476 (2018) 3478 [1802 . 02162].

[7] J. J. Condon, M. A. Condon, G. Gisler and J. J. Puschell, Strong radio sources in bright spiral galaxies. II - Rapid star formation and galaxy-galaxy interactions, ApJ 252 (1982) 102. 
[8] E. J. M. Colbert, S. A. Baum, J. F. Gallimore, C. P. O'Dea and J. A. Christensen, Large-Scale Outflows in Edge-on Seyfert Galaxies. II. Kiloparsec-Scale Radio Continuum Emission, ApJ 467 (1996) 551.

[9] J. L. Richards and M. L. Lister, Kiloparsec-Scale Jets in Three Radio-Loud Narrow-Line Seyfert 1 Galaxies, ApJL 800 (2015) L8 [1 501.05299 ].

[10] M. Berton, E. Congiu, E. Järvelä, R. Antonucci, P. Kharb, M. L. Lister et al., Radio-emitting narrow-line Seyfert 1 galaxies in the JVLA perspective, A\&A 614 (2018) A87 [1801.03519].

[11] C. G. Mundell, A. S. Wilson, J. S. Ulvestad and A. L. Roy, Parsec-Scale Images of Flat-Spectrum Radio Sources in Seyfert Galaxies, ApJ 529 (2000) 816.

[12] M. Orienti and M. A. Prieto, Radio structures of the nuclei of nearby Seyfert galaxies and the nature of the missing diffuse emission, MNRAS 401 (2010) 2599 [0911.0852].

[13] L. C. Ho, Nuclear Activity in Nearby Galaxies, ARA\&A 46 (2008) 475 [08 03 . 2268].

[14] A. L. Roy, J. S. Ulvestad, A. S. Wilson, E. J. M. Colbert, C. G. Mundell, J. M. Wrobel et al., Free-Free Absorption on Parsec Scales in Seyfert Galaxies, in Perspectives on Radio Astronomy: Science with Large Antenna Arrays (M. P. van Haarlem, ed.), p. 173, 2000, astro-ph/9909105.

[15] P. Kharb, M. L. Lister and N. J. Cooper, Extended Radio Emission in MOJAVE Blazars: Challenges to Unification, ApJ 710 (2010) 764 [1001.0731].

[16] P. Bontempi, M. Giroletti, F. Panessa, M. Orienti and A. Doi, Physical properties of the nuclear region in Seyfert galaxies derived from observations with the European VLBI Network, MNRAS 426 (2012) 588 [1208.0230].

[17] H. Falcke, N. M. Nagar, A. S. Wilson and J. S. Ulvestad, Radio Sources in Low-Luminosity Active Galactic Nuclei. II. Very Long Baseline Interferometry Detections of Compact Radio Cores and Jets in a Sample of LINERs, ApJ 542 (2000) 197 [astro-ph/ 0005383 ].

[18] E. Middelberg, I. Agudo, A. L. Roy and T. P. Krichbaum, Jet-cloud collisions in the jet of the Seyfert galaxy NGC3079, MNRAS 377 (2007) 731 [astro-ph / 0702481 ].

[19] F. Panessa and M. Giroletti, Sub-parsec radio cores in nearby Seyfert galaxies, MNRAS 432 (2013) 1138 [1304.0794].

[20] D. V. Lal, P. Shastri and D. C. Gabuzda, Milliarcsec-scale radio structure of a matched sample of Seyfert 1 and Seyfert 2 galaxies, A\&A 425 (2004) 99 [astro-ph/ 0406597 ].

[21] K. Hada, A. Doi, H. Nagai, M. Inoue, M. Honma, M. Giroletti et al., Evidence for a Nuclear Radio Jet and its Structure down to lsim100 Schwarzschild Radii in the Center of the Sombrero Galaxy (M 104, NGC 4594), ApJ 779 (2013) 6 [1310 . 0488 ].

[22] S. Veilleux, R. B. Tully and J. Bland-Hawthorn, On the origin of the Z-shaped narrow-line region in the Seyfert galaxy NGC 3516, AJ 105 (1993) 1318.

[23] E. Middelberg, T. P. Krichbaum, A. L. Roy, A. Witzel and J. A. Zensus, Approaching NGC 3079 with VLBI, in Future Directions in High Resolution Astronomy (J. Romney and M. Reid, eds.), vol. 340 of Astronomical Society of the Pacific Conference Series, p. 140, Dec., 2005.

[24] M. J. Kukula, A. J. Holloway, A. Pedlar, J. Meaburn, J. A. Lopez, D. J. Axon et al., Unusual radio and optical structures in the Seyfert galaxy Markarian 6, MNRAS 280 (1996) 1283.

[25] P. Kharb, C. P. O’Dea, S. A. Baum, E. J. M. Colbert and C. Xu, A Radio Study of the Seyfert 
Galaxy Markarian 6: Implications for Seyfert Life Cycles, ApJ 652 (2006) 177 [astro-ph/0607603].

[26] P. Kharb, C. P. O’Dea, S. A. Baum, M. J. Hardcastle, D. Dicken, J. H. Croston et al., Very Large Baseline Array observations of Mrk 6: probing the jet-lobe connection, MNRAS 440 (2014) 2976 [1402.7174].

[27] R. M. Hjellming and K. J. Johnston, An analysis of the proper motions of SS 433 radio jets, ApJL 246 (1981) L141.

[28] J. A. Irwin and D. J. Saikia, Giant Metrewave Radio Telescope observations of NGC 3079, MNRAS 346 (2003) 977 [astro-ph / 0309373 ].

[29] P. T. Kondratko, L. J. Greenhill and J. M. Moran, Evidence for a Geometrically Thick Self-Gravitating Accretion Disk in NGC 3079, ApJ 618 (2005) 618 [astro-ph/0408549].

[30] D. Mukherjee, G. V. Bicknell, A. Y. Wagner, R. S. Sutherland and J. Silk, Relativistic jet feedback III: feedback on gas disks, MNRAS, arXiv 1803.08305 (2018) [1803.08305].

[31] A. Brunthaler, H. Falcke, G. C. Bower, M. F. Aller, H. D. Aller and H. Teräsranta, The extreme flare in III Zw 2:. Evolution of a radio jet in a Seyfert galaxy, A\&A 435 (2005) 497.

[32] I. Martí-Vidal, J. M. Marcaide, A. Alberdi, M. A. Pérez-Torres, E. Ros and J. C. Guirado, Detection of jet precession in the active nucleus of M 81, A\&A 533 (2011) A111 [1107.0704].

[33] J. E. Conway and D. W. Murphy, Helical jets and the misalignment distribution for core-dominated radio sources, ApJ 411 (1993) 89.

[34] D. J. Saikia and M. Jamrozy, Recurrent activity in Active Galactic Nuclei, Bulletin of the Astronomical Society of India 37 (2009) [1002.1841].

[35] J. A. Irwin, P. Schmidt, A. Damas-Segovia, R. Beck, J. English, G. Heald et al., CHANG-ES - VIII. Uncovering hidden AGN activity in radio polarization, MNRAS 464 (2017) 1333 [1609.07609].

[36] J.-M. Wang, Y.-M. Chen, C. Hu, W.-M. Mao, S. Zhang and W.-H. Bian, Active Galactic Nuclei with Double-Peaked Narrow Lines: Are they Dual Active Galactic Nuclei?, ApJL 705 (2009) L76 [0 910 . 0580].

[37] P. Kharb, M. Das, Z. Paragi, S. Subramanian and L. P. Chitta, VLBI Imaging of the Double Peaked Emission Line Seyfert KISSR 1494, ApJ 799 (2015) 161 [1412. 0400 ].

[38] P. Kharb, S. Subramanian, S. Vaddi, M. Das and Z. Paragi, Double-peaked Emission Lines Due to a Radio Outflow in KISSR 1219, ApJ 846 (2017) 12 [1708. 00618 ].

[39] K. Rubinur, M. Das, P. Kharb and M. Honey, A candidate dual AGN in a double-peaked emission-line galaxy with precessing radio jets, MNRAS 465 (2017) 4772.

[40] K. Rubinur, M. Das and P. Kharb, Searching for dual active galactic nuclei, Journal of Astrophysics and Astronomy 39 (2018) 8 [1801.03451].

[41] P. Kharb, D. V. Lal and D. Merritt, A candidate sub-parsec binary black hole in the Seyfert galaxy NGC 7674, Nature Astronomy 1 (2017) 727 [1709. 06258].

[42] E. Momjian, J. D. Romney, C. L. Carilli and T. H. Troland, Sensitive VLBI Continuum and H I Absorption Observations of NGC 7674: First Scientific Observations with the Combined Array VLBA, VLA, and Arecibo, ApJ 597 (2003) 809. 